\title{
Post-Traumatic Stress Disorder among Emergency Medical Services Personnel: A Cross-sectional Study
}

\author{
Meshal K. Alaqeel ${ }^{1}$, Nawfal A Aljerian², Muhannad A. AlNahdi ${ }^{3}$, Raiyan Y Almaini ${ }^{4}$ \\ ${ }^{1}$ Consultant and Assistant Professor of Psychiatry College of Medicine, King Saud Bin Abdulaziz University for Health \\ Sciences, ${ }^{2}$ Consultant and Associate Professor Emergency Medicine College of Medicine, King Saud Bin Abdulaziz \\ University for Health Sciences, ${ }^{3,4}$ Medical Student College of Medicine, King Saud Bin Abdulaziz University for Health \\ Sciences
}

Background: Post-Traumatic Stress Disorder is characterized by directly experiencing traumatic events or witnessing, learning, or repetitively experiencing the details of these events. High risk occupations (i.e., EMS personnel) frequently encounter traumatic eventscoping with events exceeding the range of human experiences. Regional lack of studies necessitated the need to report the prevalence of PTSD among healthcare providers in Saudi Arabia (SA). Aims and Objective: To explore the prevalence of PTSD among EMS personnel Materials and Methods: This a descriptive cross-sectional study at King Abdul Aziz Medical City (KAMC), Riyadh, SA among 110 paramedics, emergency medical technicians (EMT), and ambulance drivers who were conveniently selected for this screening. Post-Traumatic Stress Disorder Checklist, the Civilian version, (PCL-C) was used to screen the participants, and cut-off point was 30-35. PTSD symptoms (present \& absent) was considered as outcome measure. Results: Total of 110 participants were contacted, $74(67 \%)$ agreed to participate. Around half of the participants were EMT (52\%). Total number of screened individuals is 52 , $14(26.9 \%)$ had positive screening, of those, EMT had higher proportion than paramedics (32\% vs $20.8 \%$ ). Conclusion: This report is the first PTSD prevalence among healthcare providers in SA. The results showed that $26 \%$ of EMS personnel had positive screening for PTSD. Attention should be directed toward this issue by regular psychological evaluation and implementing psychological rehabilitation programs for EMS personnel. The study reflects only one center in the city, thus generalization would not be inappropriate

Key words: Emergency medicine Post-traumatic Stress disorder, Saudi arabia

\section{INTRODUCTION}

Post-traumatic stress disorder (PTSD) was first recognized as a disorder in 1980 and was included in the Diagnostic and Statistical Manual of Mental Disorders (DSM) $3^{\text {rd }}$ edition. ${ }^{1}$ According to DSM $5^{\text {th }}$ edition, PTSD is characterized by exposure to traumatic events (such as car accidents, violence, and rape), either through direct experience, witnessing events, learning of such events occurring to close friends or relatives, or repetitively experiencing the details of these events. ${ }^{2}$ Moreover, this is combined with symptoms associated with traumatic events, such as intrusive dreams or memories, avoidance, and changes in mood, arousal, and reactivity. Symptoms should persist more than a month for a diagnosis of PTSD rather than Acute Stress Disorder. ${ }^{2}$ Factors that increase the risk of PTSD are as follows:(I) pre-traumatic factors such as genetic, cultural factors, and prior psychiatric disorders;(II) peri-traumatic factors that include the severity of trauma, threat to life, and personal injury; and (III) post- traumatic factors such as poor coping strategies, frequent exposure, and social support. ${ }^{2}$ Interestingly, emotional support has been shown to be protective against developing PTSD. ${ }^{3,4}$ The prevalence worldwide was $10 \%$ in $2012 .{ }^{5}$ In USA, the 
prevalence is about $8.7 \%$, while the prevalence in European, Asian, and African countries is lower. ${ }^{2,5}$ Nevertheless, the prevalence differs based on occupations involving high levels of stress. ${ }^{2}$

Occupations such as police officers, emergency medical service (EMS) personnel, and fire fighters are associated with increased exposure to traumatic events, having to cope with events exceeding the range of typical human experience, suggesting a higher susceptibility for developing PTSD symptoms. ${ }^{2,6}$ EMS personnel are at a higher risk than are other emergency personnel. ${ }^{5}$ The repetition of these events cumulatively and negatively affect their well-being, making them more vulnerable; however, normally where no repetitive traumatic events occur, symptoms may fade away with time. ${ }^{7,8}$

The prevalence of PTSD among high-risk occupations has been shown to be high, ranging from $3 \%$ to $24.5 \%{ }^{9,10}$ The prevalence among EMS personnel is up to $22 \%$ in several studies. ${ }^{9}{ }^{10}$ In Sweden, a study surveyed362EMS personnel and 223 reported having traumatic events. In this study, the occurrence of symptoms among EMS personnel reached $15 \%$ based on the Impact of Event Scale and 12\% based on the PTSD-10 scale. ${ }^{6}$ However, the general population had a lower percentage. ${ }^{6}$ Other studies contradict the findings of the preceding ones, stating that the prevalence is lower compared to other studies, at approximately $6 \%$. This sole finding may be due to the delay in the assessment of the workers, as the study suggested. ${ }^{11}$

In Saudi Arabia (SA), among high risk groups, only firefighters were screened for PTSD. ${ }^{12}$ The deficit in the literature was evident, necessitating a study to evaluate the prevalence of PTSD among healthcare workers, particularly EMS personnel. Therefore, this study aims to identify the prevalence of PTSD among EMS personnel in SA.

\section{MATERIALS AND METHODS}

This descriptive cross-sectional study was based in King Abdulaziz Medical City (KAMC), Riyadh, SA. KAMC is a tertiary health care center and considered a level I trauma center, providing widespread 24-hour daily trauma care, ensuring proper management by different specialties. Additionally, KAMC responds to pre-hospital emergency calls in its catchment area via its own EMS department.

The study targeted all paramedics, emergency medical technicians (EMTs), and drivers in KAMC. The EMS management office provided contact details for 110 employees under its supervision. Study subjects were conveniently selected and approached through their email provided by the EMS department. Each participant received an invitation to participate in the study. The purpose and objectives were explained and their voluntary enrollment was obtained. A link was attached to participate in the study and fill the designed questionnaire. All data were anonymized and analyzed with no possibility of identification.

The questionnaire used in this study was adopted after obtaining the approval of the National PTSD center. The first part examined demographic variables, such as age, marital status, position, and previous psychiatric history or consultations. The second used the Post-Traumatic Stress Disorder Checklist, the Civilian version (PCL-C). The PCL-C is composed of 17 self-reported questions designed for screening and helping in the diagnosis of PTSD based on the criteria in DSM-IV using a 5-point Likert scale. The total score for Likert scale responses ranged 17-85, with 1 representing not at all to 5 representing extremely. The data were collected in a 6-week period. This study was reviewed and approved by the Institutional Review Board (IRB) at King Abdullah International Medical Research center (KAIMRC).

\section{Statistical analysis}

The data were imported into Microsoft Excel 2016 Version 15.25 (Microsoft Corporation, Redmond, WA, USA) and analyzed using SPSS Version 24 (IBM Corp. Armonk, NY, USA). Categorical variables are presented as percentage and frequency. For the outcome variable of PTSD status, a cut-off point of 30-35 was used to consider the presence of PTSD symptoms, as suggested by the National PTSD center for the general population. ${ }^{13} \mathrm{~A}$ chi-square test was used to test for an association between categorical variables and PTSD (present and absent). A p-value of 0.05 was considered statistically significant and a 95\% confidence interval was used to report the precision of the prevalence.

\section{RESULTS}

A total of 110 EMS personnel were contacted to be enrolled in study, $74(67.3 \%)$ agreed to participate. All participants were men; $40(54 \%)$ were older than 30years and $42(70 \%)$ were married. Around half of the participants were EMTs $(39,52.7 \%)$. The majority had worked for less than 10 years $(39,65 \%$ ) (Table 1). The total number of screened individuals was 52 and 14 (26.9\%) (95\% CI: $15.6-41 \%$ ) participants scored at or above the cut-off point. Of those, EMTs formed a higher proportion than paramedics (8 [32\%] and $5[0.8 \%]$, respectively). However, of all participants, 10 (13.5\%) (95\% CI: 6.6\% to $23.4 \%$ ) had already been diagnosed with PTSD (Table 2). Unexpectedly, PTSD status did not have a statistically significant association with the socio-demographic characteristics of study subjects (Table 3 ). 


\section{DISCUSSION}

This is the first study of PTSD prevalence among healthcare providers in SA. The results show that a quarter of screened EMS personnel had positive screening for PTSD. Internationally, a systemic review showed that five out of seven studies reported that the prevalence of PTSD was over $20 \% .{ }^{14}$ Comparably, in SA, $57 \%$ of screened firefighters had positive results for PTSD. ${ }^{12}$ Previous findings show substantial differences. This might be due to the lack of outlets for firefighters to talk about their experiences, as the

\begin{tabular}{|c|c|}
\hline Characteristics & No.(\%) \\
\hline \multicolumn{2}{|c|}{ Age groups (in years) } \\
\hline$<=30$ & $34(45.9)$ \\
\hline$>30$ & $40(54.1)$ \\
\hline \multicolumn{2}{|c|}{ Duration of service (in years) $(n=60)$} \\
\hline$<=10>$ & $39(65.0)$ \\
\hline 10 & $21(35.0)$ \\
\hline \multicolumn{2}{|l|}{ Position } \\
\hline EMT & $39(52.7)$ \\
\hline Paramedic & $32(43.2)$ \\
\hline Others & $3(4.1)$ \\
\hline \multicolumn{2}{|c|}{ Related to military $(n=60)$} \\
\hline Yes & $4(6.7)$ \\
\hline No & $56(93.3)$ \\
\hline \multicolumn{2}{|c|}{ Social status $(n=60)$} \\
\hline Single & $17(28.3)$ \\
\hline Married & $42(70.0)$ \\
\hline Divorce & $1(1.7)$ \\
\hline
\end{tabular}

reports stated. ${ }^{12}$ This may not be the case for EMS personnel as they have access to healthcare facilities.

The prevalence of PTSD in the general population in SA has not yet been reported. The stigma of receiving psychological support might underlie this lack of data. ${ }^{15}$ However, in Sweden, the public had tenfold less risk of having PTSD than ambulance personnel. ${ }^{6}$ A meta-analysis of 20,000 rescuers found that the prevalence of PTSD was $10 \%$, which is close to the worldwide prevalence. ${ }^{5}$ This variability between the numbers nationally and internationally calls for more attention to resolve the consequences of PTSD. The persistence of symptoms might lead to a burden on the health care system affecting workers through chronic fatigue, decreased job satisfaction, and early retirement. ${ }^{14}$

No significant association was found between sociodemographic variables and PTSD status. Nevertheless, the data showed that married participants had half the risk of developing PTSD of single participants. Similarly, Alghamdi et al. supported this finding. They suggested that this might be due to married individuals having better chances of getting emotional support than single individuals. ${ }^{12}$ In Brazil, male ambulance workers with PTSD were more likely to be single. ${ }^{16}$ Furthermore, the findings suggest that longer duration of service is weakly associated with a lesser chance of developing PTSD symptoms. These findings concur with those in the literature as well. Employment seniority has been shown to lessen PTSD

Table. 2: Prevalence of PTSD among the study subjects

\begin{tabular}{lc} 
Prevalence & No.(\%)-(95\% Cl) \\
\hline By response to a question (suffering with PTSD) $(n=74)$ & $10(13.5 \%)-(6.67 \%$ to $23.43 \%)$ \\
By PCL - 17 item self-report DSM-IV symptoms of PTSD $(n=52)$ & $14(26.9 \%)-(15.55 \%$ to $41 \%)$ \\
\hline
\end{tabular}

\begin{tabular}{|c|c|c|c|c|}
\hline \multirow[t]{2}{*}{ Characteristics } & \multicolumn{2}{|c|}{ PTSD-No.(\%) } & \multirow[t]{2}{*}{$X^{2}$-value } & \multirow[t]{2}{*}{ p-value } \\
\hline & $\begin{array}{c}\text { Present } \\
(n=14)\end{array}$ & $\begin{array}{c}\text { Absent } \\
(n=38)\end{array}$ & & \\
\hline \multicolumn{5}{|c|}{ Age groups (in years) } \\
\hline$<=30$ & $7(28 \%)$ & $18(72 \%)$ & 0.028 & 0.866 \\
\hline$>30$ & $7(25.9 \%)$ & $20(74.1 \%)$ & & \\
\hline \multicolumn{5}{|c|}{ Duration of service (in years) $(n=51)$} \\
\hline$<=10$ & $10(32.3 \%)$ & $21(67.7 \%)$ & 0.917 & 0.338 \\
\hline$>10$ & $4(20 \%)$ & $16(80 \%)$ & & \\
\hline \multicolumn{5}{|l|}{ Position } \\
\hline EMT & $8(32 \%)$ & $17(68 \%)$ & 0.843 & 0.656 \\
\hline Paramedic & $5(20.8 \%)$ & $19(79.2 \%)$ & & \\
\hline Others & $1(33.3 \%)$ & $2(66.7 \%)$ & & \\
\hline \multicolumn{5}{|l|}{ Related to military } \\
\hline Yes & $1(33.3 \%)$ & $2(66.7 \%)$ & -- & $0.618^{*}$ \\
\hline No & $13(26.5 \%)$ & $36(73.5 \%)$ & & \\
\hline \multicolumn{5}{|c|}{ Social status $(n=51)$} \\
\hline Single & $6(40 \%)$ & $9(60 \%)$ & 1.680 & 0.195 \\
\hline Married & $8(22.2 \%)$ & $28(77.8 \%)$ & & \\
\hline
\end{tabular}


symptoms, and fewer years of experience, poor social support, and degree of stress exposure predicted higher symptoms when stressor was controlled. ${ }^{14,17}$

EMS personnel were affected more than paramedics, supporting the role of education as a risk factor, as a metaanalysis found that educational difference in one of risk factors for developing PTSD. ${ }^{3}$ Further researches are needed to understand the risk factors and current coping mechanisms in the population. Additionally, assigning a psychiatric assistant, and implementing rehabilitation programs is pivotal to tackling and lessening the disorder's burden.

The high prevalence may be due to the small sample size relative to other international studies; however, the response rate reached $67 \%$ which is very good for internet survey. Nevertheless, this reflects a true rise in the prevalence of PTSD, in accordance with another national report. The screening was performed using a self-reported questionnaire subject to participant bias. Follow-up consultation will use a more reliable assessment.

\section{Limitations}

Although this study reflects only one center in the city, thus generalization would be partly inappropriate, it directs attention towards this issue. A future direction is the performance of a nationwide study yielding conclusive numbers and an accurate estimate of prevalence.

\section{CONCLUSION}

In conclusion, this study found an alarmingly high PTSD prevalence of $26.9 \%$ relative to findings from international studies. This calls for attention towards further exploration and accurately establishing prevalence, risk factors, and coping mechanisms among the population and, thereafter, implementing regular psychiatric assistance and psychological rehabilitation programs.

\section{REFERENCES}

1. Friedman MJ. PTSD history and overview. United States Department of Veterans Affairs. 2007.
2. American Psychiatric Association. Diagnostic and statistical manual of mental disorders (DSM-5®). Am Psychiatr Publ 2013; 271-280.

3. Brewin CR, Andrews B and Valentine JD. Meta-analysis of risk factors for posttraumatic stress disorder in trauma-exposed adults. J Consult Clin Psychol 2000;68(5):748-766.

4. Ozer EJ, Best SR, Lipsey TL and Weiss DS. Predictors of posttraumatic stress disorder and symptoms in adults: a metaanalysis. Psychol Bull 2003; 129(1):52-73.

5. Berger W, Coutinho ES, Figueira I, Marques-Portella C, Luz MP, Neylan TC, et al. Rescuers at risk: a systematic review and meta-regression analysis of the worldwide current prevalence and correlates of PTSD in rescue workers. Soc Psychiatry Psychiatr Epidemiol 2012; 47(6):1001-1011.

6. Jonsson A, Segesten K and Mattsson B. Post-traumatic stress among Swedish ambulance personnel. J Emerg Med 2003; 20(1):79-84.

7. Friedman MJ, Keane TM, Resick PA, editors. Handbook of PTSD: Science and practice. $2^{\text {nd }}$ ed. Guilford Press; 2014:737.

8. Van Der Ploeg E and Kleber RJ. Acute and chronic job stressors among ambulance personnel: predictors of health symptoms. Occup Environ Med 2003; 60(suppl 1):40-46.

9. Andersen HS, Christensen AK and Petersen GO. Post-traumatic stress reactions amongst rescue workers after a major rail accident. Anxiety Stress Coping1991; 4(3):245-251.

10. Clohessy $S$ and Ehlers A. PTSD symptoms, response to intrusive memories and coping in ambulance service workers. $\mathrm{Br} \mathrm{J}$ Clin Psychol 1999;38(3):251-265.

11. Misra M, Greenberg N, Hutchinson C, Brain A and Glozier N. Psychological impact upon London Ambulance Service of the 2005 bombings. Occup Med (Lond) 2009;59(6):428-433.

12. Alghamdi $\mathrm{M}$, Hunt $\mathrm{N}$ and Thomas $\mathrm{S}$. Prevalence rate of PTSD, Depression and Anxiety symptoms among Saudi Firefighters. J Trauma Stress Disord Treat 2016;6(1):1-6.

13. PTSD Checklist (PCL). U. S Departement of Veterans Affairs D, Health Administration V, Health Strategic Healthcare Group M, Center for PTSD N. Available from: http://sph.umd.edu/sites/ default/files/files/PTSDChecklistScoring.pdf.

14. Sterud T, Ekeberg $\varnothing$ and Hem E. Health status in the ambulance services: a systematic review. BMC Health Serv Res 2006;6(1):82.

15. Wahass $S$ and Kent $G$. The modification of psychological interventions for persistent auditory hallucinations to an Islamic culture. Behav Cognit Psychother 1997; 25(4):351-364.

16. Berger W, Figueira I, Maurat AM, Bucassio EP, Vieira I, Jardim SR, et al. Partial and full PTSD in Brazilian ambulance workers: prevalence and impact on health and on quality of life. J Trauma Stress 2007; 20(4):637-642.

17. Mealer M, Burnham EL, Goode CJ, Rothbaum B and Moss M. The prevalence and impact of post traumatic stress disorder and burnout syndrome in nurses. Depress Anxiety 2009; 26(12):1118-1126.

\footnotetext{
Authors Contribution:

MKA - Concept and designing of the study, statistical analysis, manuscript preparation; NAA - Critical revision of manuscript and review of the study; MN - Reviewed the literature and data collected; RYA - Data collected and statistical analysis

Work attributed to:

Department of Psychiatry, College of Medicine, King Saud bin Abdulaziz University for Health Sciences, Riyadh, Saudi Arabia.

Orcid ID:

Dr.Meshal K Alaqeel- (D) http://orcid.org/0000-0002-3786-334X

Dr.Nawfal A Aljerian- (1) https://orcid.org/0000-0002-3242-0312

Muhannad A AINahdi- (D) https://orcid.org/0000-0003-0839-4131

Raiyan Y Almaini- (1) https://orcid.org/0000-0002-8613-0222

Source of Support: Nil, Conflict of Interest: None declared.
} 